\title{
Localization of Collagen Type IV Alpha Chains at the Basement Membrane of Oral Squamous Cell Carcinoma
}

\author{
Ryo Tamamura ${ }^{1)}$, Hitoshi Nagatsuka ${ }^{1)}$, Mehmet Gunduz ${ }^{1)}$, Hong Gong Liuº, ${ }^{2)}$ Noriyuki Nagai ${ }^{1}$ \\ 1) Okayama University, Graduate School of Medicine, Dentistry and Pharmaceutical Sciences \\ 2) Capital Medical University, Department of Pathology
}

\begin{abstract}
Destruction of basement membrane is an important element in invasion of cancer cells. Type IV collagen, the major component of basement membrane has six distinct $\alpha$ chains. In this study, we investigated the localization of six $\alpha$ chains and matrix metalloproteinases (MMPs) in oral squamous cell carcinoma immunohistochemically. In well differentiated squamous cell carcinoma, the localization of $\alpha$ chains showed various patterns. $\alpha 1$ (IV), $\alpha 2$ (IV), $\alpha 5$ (IV) and $\alpha 6$ (IV) chains were stained in almost all basement membranes in the central of cancer. Unique staining with only $\alpha 5$ (IV) and $\alpha 6$ (IV) chains was also observed. In the invasive point of cancer, all a chains mainly became negative, but MMP-2 and MMP-9 were stained strongly. In poorly differentiated squamous cell carcinoma, both a chains and MMPs were not stained. Furthermore, we examined the correlation between distribution of $\alpha 1$ chain and invasive pattern of cancer. We found that the disappearance of $\alpha$ chains correlated closely with invasive activity of cancer.
\end{abstract}

\section{Introduction}

Destruction of basement membrane is an important element in the invasion of cancer cells. Type IV collagen, the major component of the basement membrane has six distinct $\alpha$ chains. In this study, we investigated the localization of six $\alpha$ chains and matrix metalloproteinases (MMPs) in oral squamous cell carcinoma immunohistochemically.

\section{Materials and Methods}

\section{Specimens}

Folmalin-fixed, paraffin embedded sections from 30 oral squamous cell carcinomas (20 well and moderately differentiated squamous cell carcinomas and 10 poorly differentiated squamous cell carcinomas) were obtained from archival paraffin blocks at Okayama University Hospital.

\section{Immunohistochemistry}

Sections of $4 \mathrm{um}$ in thickness were dewaxed in xylene and rehydrated in alcohol, then heated to $121^{\circ} \mathrm{C}$ in an autoclave for 5 minutes. The endogenous peroxidase activity was suppressed by a solution of $0.3 \%$ hydrogen peroxide in methanol for 30 minutes. After being rinsed 3 times in Tris-buffered saline (TBS), the sections were treated for 18 hours with rat monoclonal antibodies ( $\alpha 1-6$, Shigei Medical Research Institute, Okayama, Japan) and mouse monoclonal antibodies (MMP-2, -9, Fuji Chemical, Toyama, Japan) at $4^{\circ} \mathrm{C}$. After washing three times in TBS, the
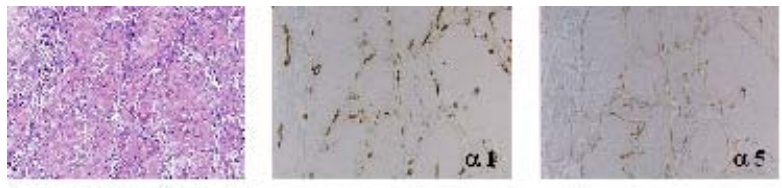

Fig.1
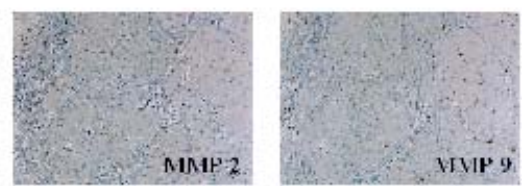

sections were treated with ABC kit (Vector Lab.) for 30 minutes at room temperature (RT). The slides were then washed in TBS and developed in $0.05 \mathrm{M}$ tris $\mathrm{HCl}$ (pH 7.6) containing $0.01 \% 3-3^{\prime}$ diaminobenzidine at RT. The sections were counterstained in Mayer's hematoxylin and mounted.

\section{Result}

Well differentiated squamous cell carcinoma

In H. E. section, we could see many islands of cancer nests with keratinization of cancer cells.

$\alpha 1$ and $\alpha 5$ chains were stained in almost all basement membranes in the central part of the cancer. ( $\alpha 2$ and $\alpha 6$ chains are not shown) MMP-2 and MMP-9 were not stained.

In well differentiated squamous cell carcinoma, the localization of $\alpha$ chains showed various patterns. $\alpha 1$ chain was stained partially, but $\alpha 5$ chain was stained continuously.

Moderately differentiated squamous cell carcinoma

In H. E. section we could see cord-like nests at the invasion point of cancer. The boundary between cancer and stroma was not clear. All a chains became negative, but MMP-2 and MMP-9 were stained strongly in cancer cells and stromal cells.

\section{Poorly differentiated squamous cell carcinoma}

In H. E., cancer cells with strong atypia proliferated solidly.

All a chains were not stained. a1 and $\alpha 2$ chains were stained only around the vessels.

Though MMP-9 were stained partially, almost all MMP's were not stained.

\section{Conclusion}

Fig.2
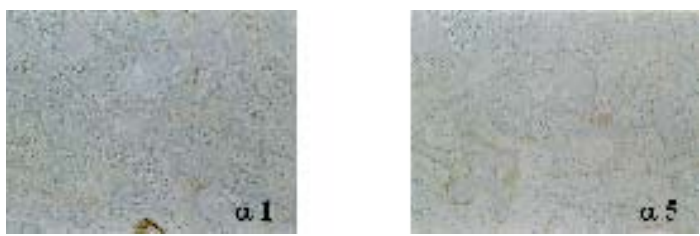
J.Hard Tissue Biology.14(2)Proceeding,2005

In oral squamous cell carcinoma (SCC), the localization of a chains is closely related to the differentiation level of the cancer. Well and moderately differentiated SCC has the ability of forming $\alpha$ chains more than poorly differentiated SCC.

In the invasion point of well and moderately differentiated SCC $\alpha$ chains were resolved by MMPs, while in poorly differentiated
SCC, no a chain was formed. The absence of $\alpha$ chains is of great importance in the invasion of cancer cells.

Moreover, we could observe the unique localization of a chain which is only composed of $\alpha 5$ and $\alpha 6$ chains. This abnormal pattern of $\alpha$ chains was made by the cancer cells.
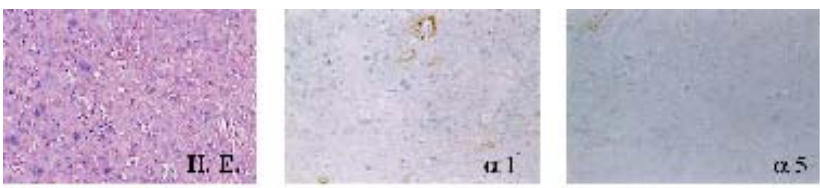

fig.4
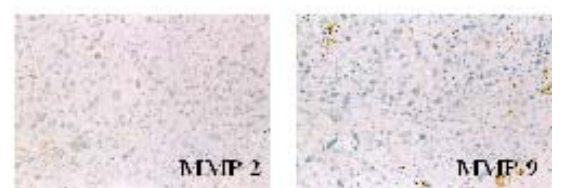